\section{Chemical Synthesis of PEDOT Nanotubes}

\author{
Xinyu Zhang, ${ }^{\dagger}$ Jeong-Soo Lee, ${ }^{\ddagger}$ Gil S. Lee, ${ }^{\ddagger}$ \\ Dong-Kyu Cha, ${ }^{\dagger}$ Moon J. Kim,,${ }^{\ddagger}$ Duck J. Yang, ${ }^{\dagger}$ and \\ Sanjeev K. Manohar*,
}

Alan G. MacDiarmid Center for Innovation, Department of Chemistry and Department of Electrical Engineering, The University of Texas at Dallas, Richardson, Texas 75080

Received September 9, 2005

Revised Manuscript Received December 2, 2005

We describe a rapid, room temperature, reverse emulsion polymerization method to chemically synthesize bulk quantities of micrometers long nanotubes of electrically conducting poly(3,4-ethylenedioxythiophene) (PEDOT) having tube diameters in the range 50-100 $\mathrm{nm}$. Composites of PEDOT nanotubes with noble metals, metal oxides, etc., can be readily synthesized using postsynthesis and in situ polymerization methods.

In addition to its environmental stability and low redox potential, poly(3,4-ethylenedioxythiophene) (PEDOT) is unique among conducting polymers in that its small band gap confers high optical transparency in the doped, conducting state. ${ }^{1}$ Although it has been extensively investigated for use in antistatic coatings, flexible electronic devices, and transparent electronics, ${ }^{2}$ its 1D transport properties have been largely unexplored because powders and films of PEDOT obtained possess granular or particulate morphology. Although nanotubes of PEDOT have been electrochemically synthesized in the pores of aluminum oxide template, ${ }^{3}$ a convenient chemical synthetic route to the bulk quantities of PEDOT nanotubes/fibers has remained a challenge. The PEDOT system is particularly recalcitrant to fibrillar or tubular polymer growth, and techniques such as nanofiber seeding, ${ }^{4}$ activated seeding, ${ }^{5}$ and interfacial polymerization ${ }^{6}$ that have been used to synthesize nanofibers of polyaniline or polypyrrole yield only granular powders when extended to the PEDOT system. In a recent report, polypyrrole nanotubes were synthesized using a hexane/water reverse microemulsion system using sodium bis(2-ethylhexyl) sulfosuccinate (AOT) cylindrical micelles as the template and $\mathrm{FeCl}_{3}$ as the oxidant. ${ }^{7}$ When applied to the PEDOT system, however, the same system yielded nanorods and not nanotubes. ${ }^{8}$ Enhanced hydrophobicity and slow kinetics of oxidation of 3,4-ethylenedioxythiophene (EDOT) vs pyrrole were invoked as factors that favor rodlike over tubular polymer growth in the PEDOT system. ${ }^{8}$ In this study, we have used different synthetic vectors in the above system to synthesize, for the first time, $10-20 \mu \mathrm{m}$ long PEDOT nanotubes having inner diameter in the $50-100 \mathrm{~nm}$ range.

In a typical synthesis, a reverse microemulsion was first prepared by dissolving AOT $(19.12 \mathrm{mmol})$ in $70 \mathrm{~mL}$ of $n$-hexane and adding a solution of $\mathrm{FeCl}_{3}(10.0 \mathrm{mmol})$ in $1.0 \mathrm{~mL}$ of water to it. The resulting orange-colored mixture was gently stirred for $5 \mathrm{~min}$ followed by addition of EDOT monomer ( $3.52 \mathrm{mmol})$. After $3 \mathrm{~h}$ under gentle magnetic stirring, the blue/black precipitate of PEDOT nanotubes was suction filtered and washed with copious amounts of methanol and acetonitrile. Drying under dynamic vacuum for $12 \mathrm{~h}$ at $80^{\circ} \mathrm{C}$ yielded a navy blue powder having the elemental composition: $\mathrm{C}, 45.54 ; \mathrm{H}, 3.52 ; \mathrm{O}, 27.56$;

\footnotetext{
Department of Chemistry.

Department of Electrical Engineering.

* Corresponding author: Fax (+1)972-883-6586; e-mail sanjeev.manohar@utdallas.edu.
}

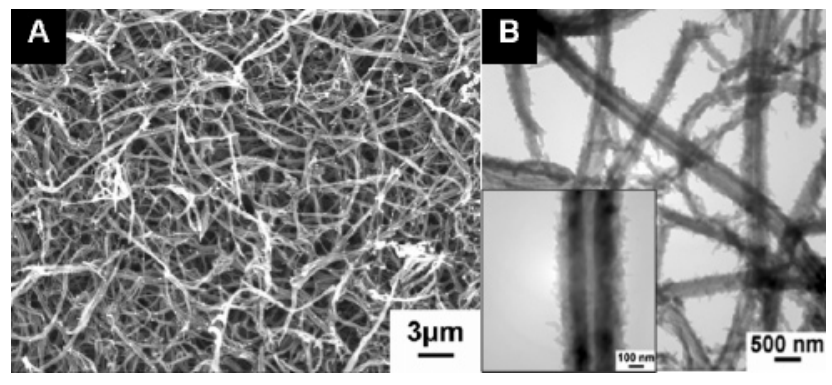

Figure 1. Microscopy images of PEDOT nanotubes: (A) SEM; (B) TEM. Inset: magnified image.

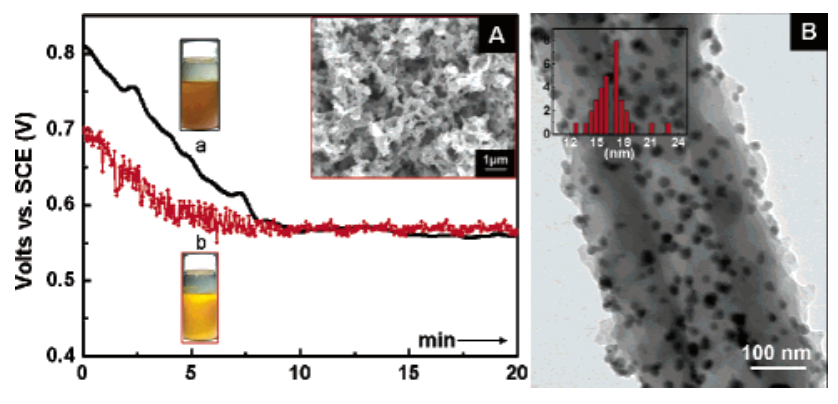

Figure 2. (A) Potential-time profile of the polymerization with EDOT added before an emulsion is obtained (plot a, orange vial) and after an emulsion is obtained (plot b, yellow vial). Inset: SEM of PEDOT stubs obtained from yellow vial. (B) Ag nanoparticles deposited on a PEDOT nanotube. Inset: Ag size distribution.

$\mathrm{Cl}, 3.19$; S, 19.66; Total: 99.47, consistent with (PEDOT)$(\mathrm{Cl})_{0.15}\left(\mathrm{H}_{2} \mathrm{O}\right)_{0.79}(>80 \%$ yield). The water in the sample could be either water of hydration or water trapped in the nanotube pore. The PEDOT nanotubes obtained exhibit a conductivity $\sigma_{\mathrm{RT}} 3-6 \mathrm{~S} / \mathrm{cm}$ (compressed pellet).

The scanning electron microscopy (SEM) and transmission electron microscopy (TEM) images show that the product is composed almost entirely of $>10 \mu \mathrm{m}$ long tubes having outer diameter in the range $300-800 \mathrm{~nm}$ and pore diameter in the range 50-100 $\mathrm{nm}$. The dramatic change in morphology from granules to tubes shows not only that the AOT/hexane reverse microemulsion method can be extended beyond the polypyrrole system $^{7}$ but also that the PEDOT system is itself very sensitive to small changes in experimental conditions. For example, the molar ratio of $\mathrm{EDOT} / \mathrm{FeCl}_{3} / \mathrm{AOT}$ in the previous study (1.0:3.47:6.14) is similar to the present study (1.0:2.84:5.43), and yet, there is a change in morphology from rods to tubes. ${ }^{9}$ This can be traced to two important differences in our synthetic procedure: (i) our reaction mixture was three times more dilute and not viscous, and (ii) the $\mathrm{AOT} / \mathrm{FeCl}_{3} /$ hexane mixture was not vigorously stirred to obtain a milky yellow emulsion prior to addition of EDOT monomer. Nanotubes are obtained if the EDOT monomer is added to a gently stirred suspension of AOT/ $\mathrm{FeCl}_{3} /$ hexane that still had the initial orange in color from the $\mathrm{FeCl}_{3}$ oxidant (Figure 2A, vial). If, however, the $\mathrm{AOT} / \mathrm{FeCl}_{3} /$ hexane mixture is vigorously stirred to yield a light-yellow milky emulsion before adding EDOT monomer like in the previous study, short PEDOT stubs/rods are obtained (Figure 2A, inset). ${ }^{9}$ The reasons for the change in morphology are unclear, although vigorous stirring could have fragmented the long cylindrical micellar templates resulting in short PEDOT nanorods. Potentialtime profiling ${ }^{10}$ of the two systems provides evidence consistent with higher oxidation potential and faster reaction rates favoring 


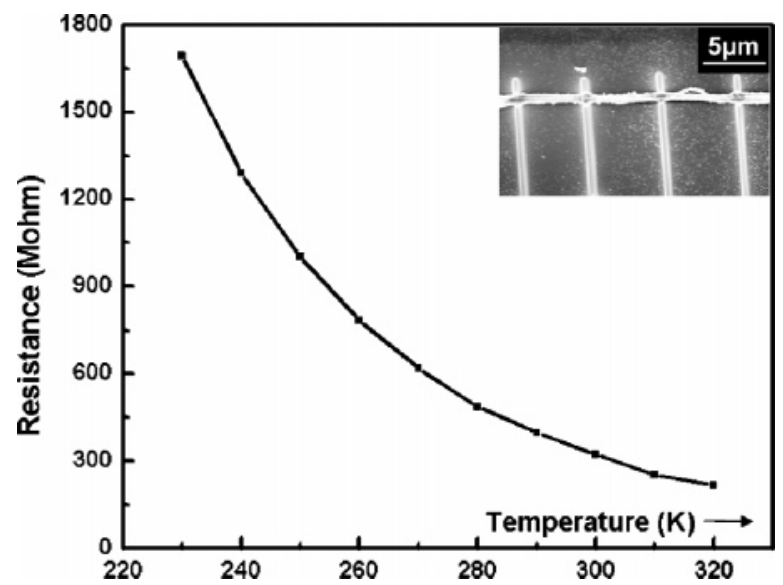

Figure 3. Resistance vs temperature plot of an individual PEDOT nanotube. Inset: SEM image showing four Pt leads deposited by FIB.

tubular polymer growth (Figure $2 \mathrm{~A}$ ). The initial oxidation potential of the orange-colored $\mathrm{AOT} / \mathrm{FeCl}_{3} /$ hexane system is $0.81 \mathrm{~V}$ (this study) is significantly higher than $0.70 \mathrm{~V}$ observed for the milky yellow emulsion (previous study). The slopes of the two plots also suggest a more rapid polymerization for the higher potential system (this study). The drop in potential from 0.81 $\mathrm{V}$ (vs SCE) in the clear orange AOT/ $/ \mathrm{FCl}_{3} /$ hexane mixture to $0.70 \mathrm{~V}$ in the milky yellow emulsion is consistent with migration of $\mathrm{Fe}^{3+}$ ions to the interior of the micelle and a slower polymerization rate. It is not clear at the present time whether tubular morphology is governed by high initial potential, or faster reaction kinetics, or both, although our working hypothesis is that while tubular growth is the preferred pathway for PEDOT in this system, the reaction rate is reduced sufficiently in the milkyyellow emulsion for secondary (nontubular) growth processes to occur. Secondary growth mechanisms have been reported to favor granular over fibrillar morphology in the polyaniline system. $^{11}$

Composites of PEDOT nanotubes and noble metals/oxides can be readily synthesized using both in-situ and postsynthesis methods. For example, when EDOT is polymerized in the presence of $100 \mathrm{mg}$ of $10-20 \mathrm{~nm}$ diameter nanospheres of magnetic iron oxide, ${ }^{9}$ a magnetic PEDOT nanotube composite was obtained having iron oxide nanoparticles randomly distributed along the outer walls and pores. The method is robust and can be extended to other metal oxides and could be important in the design of metal/polymer nanocomposites having preselected properties. Conducting PEDOT nanotubes also react with noble metal ions in solution, e.g., aqueous $\mathrm{AgNO}_{3}$, yielding 18-22 nm diameter Ag spheres uniformly distributed along the walls of the nanotubes (Figure 1B). These results are similar to the spontaneous electroless deposition of metal nanoparticles on the surface and pores of polypyrrole nanotubes when exposed to solutions of noble metal ions without any added capping or reducing agents. ${ }^{12}$ Doped PEDOT behaves analogously to doped polypyrrole and functions as a reductant in this reaction. We believe that PEDOT nanotubes are oxidized to a higher oxidation state during the reduction of $\mathrm{Ag}^{+}$ions to $\mathrm{Ag}$ nanoparticles. These noble metal nanocomposites have potential application in the design of next generation catalyst systems including vapor and analyte sensors. ${ }^{13}$

The as-synthesized, doped PEDOT nanotubes can be readily dispersed in common organic solvents and films cast on a variety of substrates. Bath sonication of $10 \mathrm{mg}$ of PEDOT nanotubes in ethanol for $10 \mathrm{~min}$ yields a homogeneous dispersion that was used to spin-cast a thin film on the surface of a $\mathrm{Si} / \mathrm{SiO}_{2}$ wafer. A single, long, individual nanotube was detected on the silicon surface using SEM, and four $0.3 \mu \mathrm{m}$ wide Pt leads, spaced 6 $\mu \mathrm{m}$ apart, were deposited on its surface using a focused ion beam system (FIB). The room temperature dc conductivity of the individual PEDOT nanotube is $\sim 10^{-3} \mathrm{~S} / \mathrm{cm}$, which is 3 orders of magnitude lower than the bulk powder. While this is significantly lower than the $7 \mathrm{~S} / \mathrm{cm}$ conductivity value obtained for HF-doped PEDOT nanotube synthesized along the pores of $\mathrm{Al}_{2} \mathrm{O}_{3}$ templates, ${ }^{3}$ it is close to the $10^{-2} \mathrm{~S} / \mathrm{cm}$ obtained when these tubes were "dedoped" with $\mathrm{NaOH}$. It is possible that PEDOT nanotubes could have been partially dedoped by the solvent (ethanol) that was used to prepare the dispersion prior to deposition on the $\mathrm{SiO}_{2}$ surface. For example, ethanol has previously been reported to dedope films of parent polythiophene grafted on polyethylene surfaces. ${ }^{14} \mathrm{We}$ expect the conductivity of these partially dedoped PEDOT nanotubes to increase upon subsequent redoping. Transport measurements on an individual PEDOT nanotube (Figure 3) show classical semiconducting behavior following a $3 \mathrm{D}$ variable range hopping (VRH) mechanism that is consistent with previous studies on individual nanofibers/tubes of polyaniline and polypyrrole. ${ }^{15}$ The metal oxide semiconductor field effect transistor (MOSFET) configuration also opens opportunities to evaluate individual PEDOT nanotubes as field effect transistors and chemical sensors (ChemFETs). . $^{3,8,16}$

In summary, we describe for the first time (i) a convenient, one-step method to chemically synthesize bulk quantities of microns long PEDOT nanotubes using reverse microemulsion polymerization, (ii) the use of potential-time profiling to uncover factors that favor tubular polymer growth, (iii) the synthesis of PEDOT/metal and PEDOT/metal oxide composites having tubular morphology, and (iv) transport properties and potential device applications of individual PEDOT tubes.

Acknowledgment. We gratefully acknowledge helpful discussions with Professor Alan G. MacDiarmid and for financial support from The University of Texas at Dallas.

Supporting Information Available: Synthetic procedure for PEDOT nanotubes and composites, device fabrication for single tube conductivity, and enlarged SEM/TEM images. This information is available free of charge via the Internet at http://pubs.acs.org.

\section{References and Notes}

(1) Groenendaal, L. B.; Jonas, F.; Freitag, D.; Pielartzik, H.; Reynolds, J. R. Adv. Mater. 2000, 12, 481. Groenendaal, L. B.; Zotti, G.; Aubert, P.-H.; Waybright, S. M.; Reynolds, J. R. Adv. Mater. 2003, 15, 855. Ha, Y.-H.; Nikolov, N.; Pollack, S. K.; Mastrangelo, J.; Martin, B. D.; Shashidhar, R. Adv. Funct. Mater. 2004, 14, 615.

(2) Hohnholz, D.; Okuzaki, H.; MacDiarmid, A. G. Adv. Funct. Mater. 2005, 15, 51. Hong, K. H.; Oh, K. W.; Kang, T. J. J. Appl. Polym. Sci. 2005, 97, 1326.

(3) Kim, B. H.; Park, D. H.; Joo, J.; Yu, S. G.; Lee, S. H. Synth. Met. 2005, 150, 279.

(4) Zhang, X.; Goux, W. J.; Manohar, S. K. J. Am. Chem. Soc. 2004 126,4502 .

(5) Zhang, X.; Manohar, S. K. J. Am. Chem. Soc. 2004, 126, 12714.

(6) Huang, J.; Kaner, R. B. J. Am. Chem. Soc. 2004, 126, 851. Zhang, X.; Chan-Yu-King, R.; Jose, A.; Manohar, S. K. Synth. Met. 2004, $145,23$.

(7) Jang, J.; Yoon, H. Chem. Commun. 2003, 720.

(8) Jang, J.; Chang, M.; Yoon, H. Adv. Mater. 2005, 17, 1616.

(9) See Supporting Information section. This includes an SEM image of PEDOT powder obtained at EDOT/ $\mathrm{FeCl}_{3} / \mathrm{AOT}$ molar ratio used in ref 8 but synthesized under dilute conditions and gentle stirring.

(10) Manohar, S. K.; MacDiarmid, A. G.; Epstein, A. J. Synth. Met. 1991, 41, 711. Wei, Y.; Hsueh, K. F.; Jang, G. W. Polymer 1994, 35, 3572.

(11) Huang, J.; Kaner, R. B. Angew. Chem., Int. Ed. 2004, 43, 5817.

(12) Zhang, X.; Manohar, S. K. J. Am. Chem. Soc. 2005, 127, 14156.

(13) Huang, J.; Virji, S.; Weiller, B. H.; Kaner, R. B. Chem.-Eur. J. 2004, 10, 1314. Smith, J. A.; Josowicz, M.; Janata, J. J. Electrochem. Soc. 2003, 150, E384. 
(14) Chanunpanich, N.; Ulman, A.; Strzhemechny, Y. M.; Schwarz, S. A.; Dormicik, J.; Janke, A.; Braun, H. G.; Kratzmuller, T. Polym. Int. 2003, 52, 172.

(15) Long, Y.; Zhang, L.; Chen, Z.; Huang, K.; Yang, Y.; Xiao, H.; Wan, M.; Jin, A.; Gu, C. Phys. Rev. B 2005, 71, 165412/165411.
(16) Lu, J.; Pinto, N. J.; MacDiarmid, A. G. J. Appl. Phys. 2002, 92, 6033.

MA051975C 